\title{
Comment on Health Literacy: The Common Denominator of Healthcare Progress
}

\author{
Halah Ibrahim ${ }^{1} \cdot$ Satish Chandrasekhar Nair ${ }^{2}$ (]) \\ Accepted: 29 September 2021 / Published online: 18 October 2021 \\ (c) The Author(s), under exclusive licence to Springer Nature Switzerland AG 2021
}

\section{Dear Editor,}

We read with great interest the commentary "Health Literacy: The Common Denominator of Healthcare Progress" by Pitts and Freeman in the September 2021 issue of The Patient [1]. We applaud the authors for highlighting the important, but often ignored, impact of health literacy on health outcomes. The World Health Organization considers health literacy to be one of the most important health indicators [2]. There is a large and growing body of literature documenting the adverse consequences of inadequate health literacy, including decreased screening and preventive services and decreased compliance with treatments [3]. This results in increased use of emergency services, increased hospitalizations, and, ultimately, higher mortality rates [3]. Despite its importance, health literacy is a global public health concern. International data confirm that many countries struggle with inadequate health literacy rates [4]. The European Health Literacy survey noted limited health literacy in $47 \%$ of respondents, inadequate health literacy in $12 \%$ of respondents, and an additional $35 \%$ with problematic health literacy [5]. In the United Arab Emirates, we found that over $60 \%$ of the population surveyed possessed inadequate health literacy [6]. A nationwide study in the US

This comment refers to the article available online at https://doi. org/10.1007/s40271-021-00537-9.

This is a reply to this letter https://doi.org/10.1007/s40271-02100557-5.

Satish Chandrasekhar Nair

schandra@seha.ae

1 Department of Medicine, Sheikh Khalifa Medical City, Abu Dhabi, United Arab Emirates

2 Department of Academic Affairs-Medical Research, Tawam Hospital Johns Hopkins Medicine International, College of Medicine and Health Sciences, UAE University, Al Ain, United Arab Emirates revealed that $36 \%$ of Americans had basic and below basic health literacy rates [7].

The coronavirus disease 2019 (COVID-19) pandemic has focused attention on the intersection between government regulations, social policy, and health outcomes. As the authors state, the disproportionately high morbidity and mortality of COVID-19 on communities of color is multifactorial and includes disinformation campaigns that targeted Black and Latinx patients, historical mistrust in government institutions, and the systemic racism that has long existed in research and healthcare, but only recently entered the public conversation on health disparities [8]. The authors suggest that health literacy should be an integral part of pandemic preparedness. In fact, as pandemic-imposed changes to the healthcare system become permanent, such as telehealth services, health inequities may continue to increase for those unable to access or use advanced technology [8].

We agree with the authors that improving health literacy should be a national priority. Programs to improve health literacy can lead to improved patient and population health outcomes. The concept of appointing a 'health literacy czar' for the US is interesting. The fear is that with the current politicization of all government agencies and roles, it may be difficult for an individual to bring forth meaningful change. A longitudinal and multi-pronged government approach is necessary, including tackling disparities in the nation's education system. Research on health inequities has consistently shown a correlation between low health literacy, decreased educational attainment, and poor health outcomes [9]. In this regard, strategies to improve health literacy may in turn improve health outcomes in patients with lower levels of education.

While government-led approaches are necessary, the healthcare community has an important role in tackling health literacy. Targeted interventions are necessary to prevent the marginalization of all patient populations, including racial and ethnic minorities, elderly patients, and those with chronic medical conditions, to ensure that they fully 
understand and are engaged with all healthcare decisions. In addition to ongoing efforts to recognize and address implicit and explicit bias in research and healthcare, individual healthcare professionals and hospitals can prioritize health literacy in daily interactions. First, healthcare providers and researchers should assess every patient's ability to understand and process the information that they are provided. This becomes especially difficult with virtual technology, where facial and body cues may not be as evident. As such, allocating appropriate time for patient visits and ensuring that all questions are answered becomes increasingly important. Integrating multilingual staff into the clinical teams, including case coordinators and patient representatives, can help overcome some language barriers. Hospitals can also design and implement professional development programs that focus specifically on using universal health literacy precautions during patient-provider interactions. Tips include avoiding medical terminology, explaining information in small, easily understandable pieces, and assessing comprehension. Using pictures and visual aids can also improve patient understanding [10]. As Pitts and Freeman state, there is no 'magic solution' for health literacy. It will indeed "take time, hard work, and commitment to convene the critical disciplines that can inform programs, practices, and metrics". As healthcare professionals, we can each do our part.

\section{Declarations}

Conflict of interest Halah Ibrahim and Satish Chandrasekhar Nair declare no conflicts of interest.
Author contributions SCN concept, design, collection of materials, and review of the manuscript. HI writing and review of the manuscript.

\section{References}

1. Pitts PJ, Freeman E. Health literacy: the common denominator of healthcare progress. Patient. 2021;14(5):455-8.

2. World Health Organization. World Health Statistics. https://www. who.int/gho/publications/world_health_statistics/EN_WHS2015_ Part2.pdf?ua=1. Accessed 16 Sept 2021.

3. Berkman ND, Sheridan SL, Donahue KE, Halpern DJ, Crotty K. Low health literacy and health outcomes: an updated systematic review. Ann Intern Med. 2011;155(2):97-107.

4. Nair SC, Satish KP, Sreedharan J, Ibrahim H. Assessing health literacy in the eastern and middle-eastern cultures. BMC Public Health. 2016;16:831.

5. Sørensen K, van den Broucke S, Fullam J, Doyle G, Pelikan J, Slonska Z, et al. Health literacy and public health: a systematic review and integration of definitions and models. BMC Public Health. 2012;12:80.

6. Nair SC, Satish KP, Sreedharan J, Muttappallymyalil J, Ibrahim $\mathrm{H}$. Improving health literacy critical to optimize telemedicine, the future of global healthcare. Telemed E-Health. 2020;26(11):1325.

7. The Health Literacy of America's Adults. https://nces.ed.gov/ pubs2006/2006483.pdf. Accessed 16 Sept 2021.

8. Scharff DP, Mathews KJ, Jackson P, et al. More than Tuskegee: understanding mistrust about research participation. J Health Care Poor Underserved. 2010;21(3):879-97.

9. van der Heide I, Rademakers J, Schipper M, Droomers M, Sørensen K, Uiters E. Health literacy of Dutch adults: a cross sectional survey. BMC Public Health. 2013;13:179.

10. Hersh L, Salzman B, Snyderman D. Health literacy in primary care practice. Am Fam Physician. 2015;92(2):118-24. 